Attribution du titre de médecin spécialiste

Verleihung des Spezialarzttitels

Conferimento del titolo di dottore specialista

\section{Umberto Costanzo}

Facharzt FMH für Dermatologie und Venerologie

Klosterstrasse 5

6003 Luzern

\section{Susanna Fistarol-Bohn}

Fachärztin FMH für Dermatologie und Venerologie

Rittergasse 29

4051 Basel

\section{André Friedli}

Médecin spécialiste FMH de dermatologie et vénéréologie

4 , rue du Pré-Naville

1207 Genève

\section{Luzius Gilli}

Facharzt FMH für Dermatologie und Venerologie

Im Reinacherhof 53

4153 Reinach

\section{Michel Grimaitre}

Médecin spécialiste FMH de dermatologie et vénéréologie

4, rue du Pré-Naville

1207 Genève

\section{Susanne Hirschel}

Médecin spécialiste FMH de dermatologie et vénéréologie

3 bis, route de Loëx

1213 Onex

\section{Pier-Paolo Pedrazzetti}

Facharzt FMH für Dermatologie und Venerologie

Zürichstrasse 34

8134 Adliswil

\section{Nouveaux cabinets médicaux Praxiseröffnungen \\ Nuovi studi medici}

\section{Umberto Costanzo}

Facharzt FMH für Dermatologie und Venerologie und Angiologie

Klosterstrasse 5

6003 Luzern

\section{Oliver Philip Kreyden}

Facharzt FMH für Dermatologie und Venerologie

Baselstrasse 9

4132 Muttenz

\section{Pier-Paolo Pedrazzetti}

Facharzt FMH für Dermatologie und Venerologie

Zürichstrasse 34

8134 Adliswil

\section{Distinction}

\section{Auszeichnung - Ehrung Onoranza}

Le Dr Albert-Adrien Ramelet, de Lausanne, a été nommé VicePrésident de l'Union internationale de phlébologie (UIP) à Rome le 12 septembre 2001. L'UIP est l'organisation faîtière mondiale de toutes les Sociétés de phlébologie.

La rédaction l'en félicite.

\section{Annonce • Ankündigung}

\section{Prix Roche 2002}

pour d'éminents travaux de recherche en dermatologie

\section{Prix}

CHF 10 000,-

Candidats

Chercheurs ou équipes de chercheurs travaillant dans des cliniques dermatologiques ou d'autres instituts universitaires

Dermatologues praticiens indépendants

\section{Propositions}

Devront être remises au président de la SSDV à l'attention du jury jusqu'au 30 avril 2002

\section{Remise du prix}

A l'occasion de la 84 e réunion annuelle de la SSDV à Berne, le 31 octobre jusqu'au 2 novembre 2002

Information

Prof. R.G. Panizzon

Chef du service de dermatologie et vénéréologie, CHUV

1011 Lausanne

\section{Roche-Preis 2002}

\section{für hervorragende Forschungsarbeiten in der Dermatologie}

\section{Preis}

\author{
CHF 10 000.-
}

\section{Teilnahmeberechtigte}

Forscher und Forscherinnen an dermatologischen Kliniken und anderen Universitätsinstituten

Frei praktizierende Dermatologen

\section{Anträge}

Einzureichen an den Präsidenten der SGDV zu Händen der Jury bis zum 30. April 2002

\section{Verleihung}

Anlässlich der 84. Jahresversammlung der SGDV in Bern vom 31. Oktober bis 2. November 2002

\section{Information}

Prof. R.G. Panizzon

Chef du service de dermatologie et vénéréologie, CHUV

1011 Lausanne 